\section{Cureus}

Received 09/24/2014

Review began 09/24/2014

Review ended 09/29/2014

Published 09/30/2014

\section{(c) Copyright 2014}

Renouf et al. This is an open access article distributed under the terms of the Creative Commons Attribution License CC-BY 3.0., which permits unrestricted use, distribution, and reproduction in any medium, provided the original author and source are credited.

\title{
Body Packing with Cocaine: A Simulation Exercise
}

Tia S. Renouf MD ${ }^{1}$, Holly Black ${ }^{2}$, Sabrina Alani ${ }^{2}$, Adam Dubrowski ${ }^{3}$

1. Emergency Medicine, Memorial University of Newfoundland/Tairawhiti Hospital, Gisborne, NZL 2. Emergency Medicine, Memorial University of Newfoundland 3. Emergency Medicine, Memorial University of Newfoundland, St. John's, CAN

$\square$ Corresponding author: Adam Dubrowski, adam.dubrowski@gmail.com Disclosures can be found in Additional Information at the end of the article

\section{Abstract}

Pedagogically sound simulation-based medical education (SBME) produces effective learning [1]. We present a simulation scenario for "body packing", which is the concealment of an illicit drug in packets (usually condoms) inside the body by swallowing or recto-vaginal insertion, usually for transportation across borders. This scenario will train Emergency Medicine residents to suspect body packing under appropriate circumstances, and to treat the potential alimentary pathologies and drug toxicities which body packing with cocaine can cause.

Categories: Emergency Medicine, Medical Education, Medical Simulation

Keywords: emergency medicine, medical education, simulation based medical education, toxicology, body packing, cocaine, hypertensive emergency, arrythmias, seizures

\section{Introduction}

Also known as "muling", body packing involves filling a body cavity with wrapped packets of an illegal drug. It is sometimes confused with "body stuffing", an evasive tactic by a person carrying generally small amounts of illicit drugs, should they feel they are about to be apprehended by police. In contrast, body packing is typically suspected at international airports, where security personnel or drug dogs are trained to find contraband. Drug packets concealed in a body cavity can leak or burst, causing a massive overdose, usually of cocaine and heroin. The packets themselves can also cause bowel or esophageal obstruction, perforation, and peritonitis. The leakage of packets containing cocaine is a medical emergency and an indication for prompt surgical removal.

For medical learners in smaller residential and urban areas, where international traffic is limited or absent, the diagnosis of body packing may be challenging. For example, trainees at Memorial University of Newfoundland (MUN) located in St. John's Newfoundland (population 208,372 as of 2013) serve a relatively small population, in which the drug trade has recently taken hold where there was once mostly petty crime. It has been projected that recent offshore gas discovery and attendant international travel may bring more cases of body packing to this province's emergency departments.

We describe a simulation exercise designed to teach trainees at MUN an approach to cocaine toxicity in the setting of body packing.

\section{Technical Report}




\section{Cureus}

This simulation session uses the Gaumard Noelle S575 human patient simulator, programmed to produce a cocaine intoxication. If requested by the learner, a plain abdominal x-ray image shows packets of drug within the bowel of a patient who is in handcuffs. Two confederates are employed as prison personnel. The detailed stepwise scenario is written and given to technical staff, who then programs the computer to control the simulation.

\section{Pre-briefing}

Before the simulation begins, students are introduced to the "fictional contract", in which they acknowledge that although the case is an invention, participants will behave as though it is real. This creates a safe place where trainees can make mistakes. It is acknowledged to be a strictly formative exercise. In this case, the abdominal x-ray image used is also a simulation. One image, obtained online, shows evidence of body packing (Figure 1), while the other, because of an error in positioning a patient in our x-ray department, shows an inmate/patient's handcuffs and shackles in an otherwise unremarkable film (Figure 2). To help tell the story, two real x-ray images were superimposed (Figure 3). The students are told, "this hybrid image is obviously not real, but we would like you to suspend your disbelief for the purposes of learning". 


\section{Cureus}

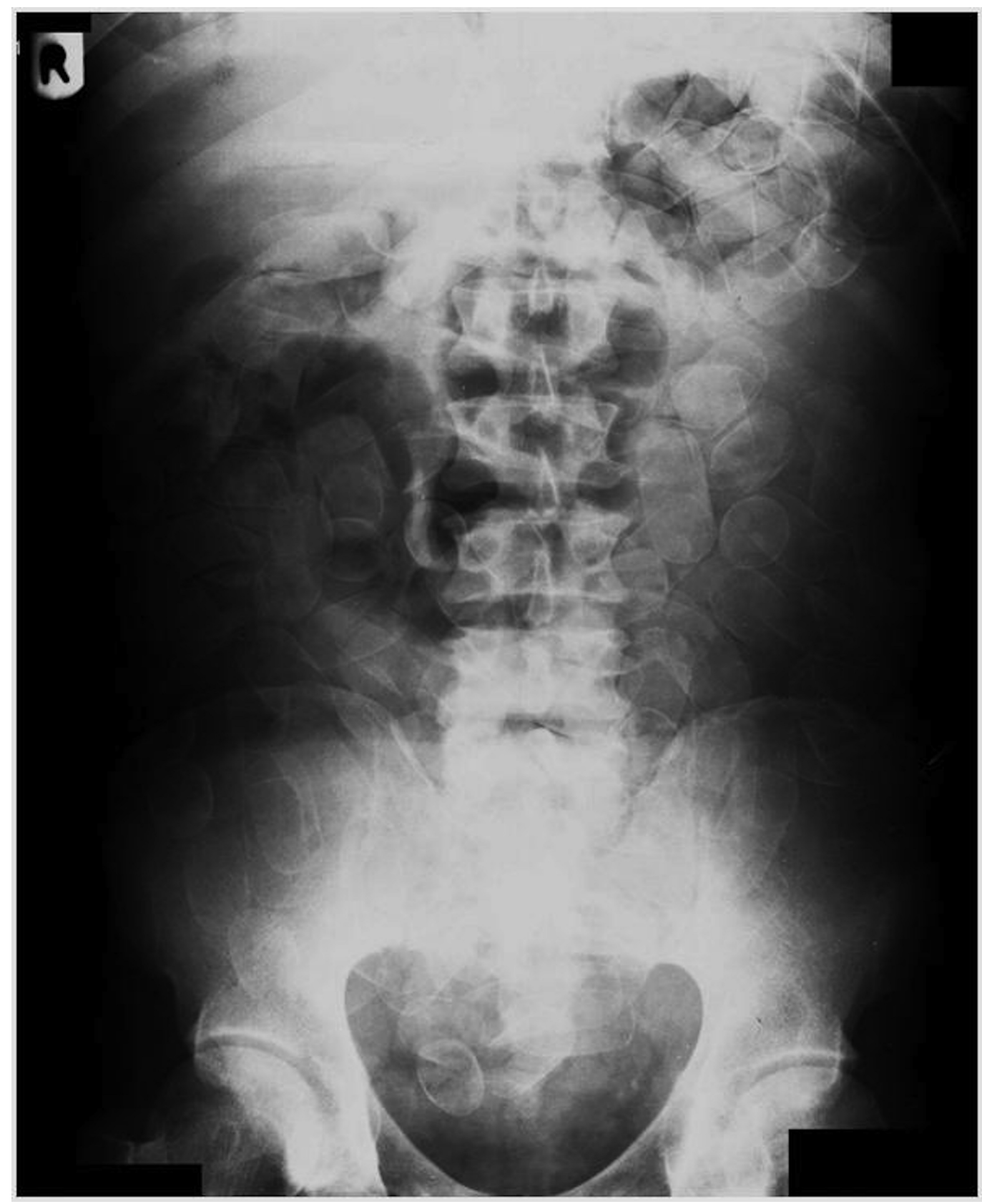

FIGURE 1: Abdominal x-ray image showing packets of cocaine 


\section{Cureus}

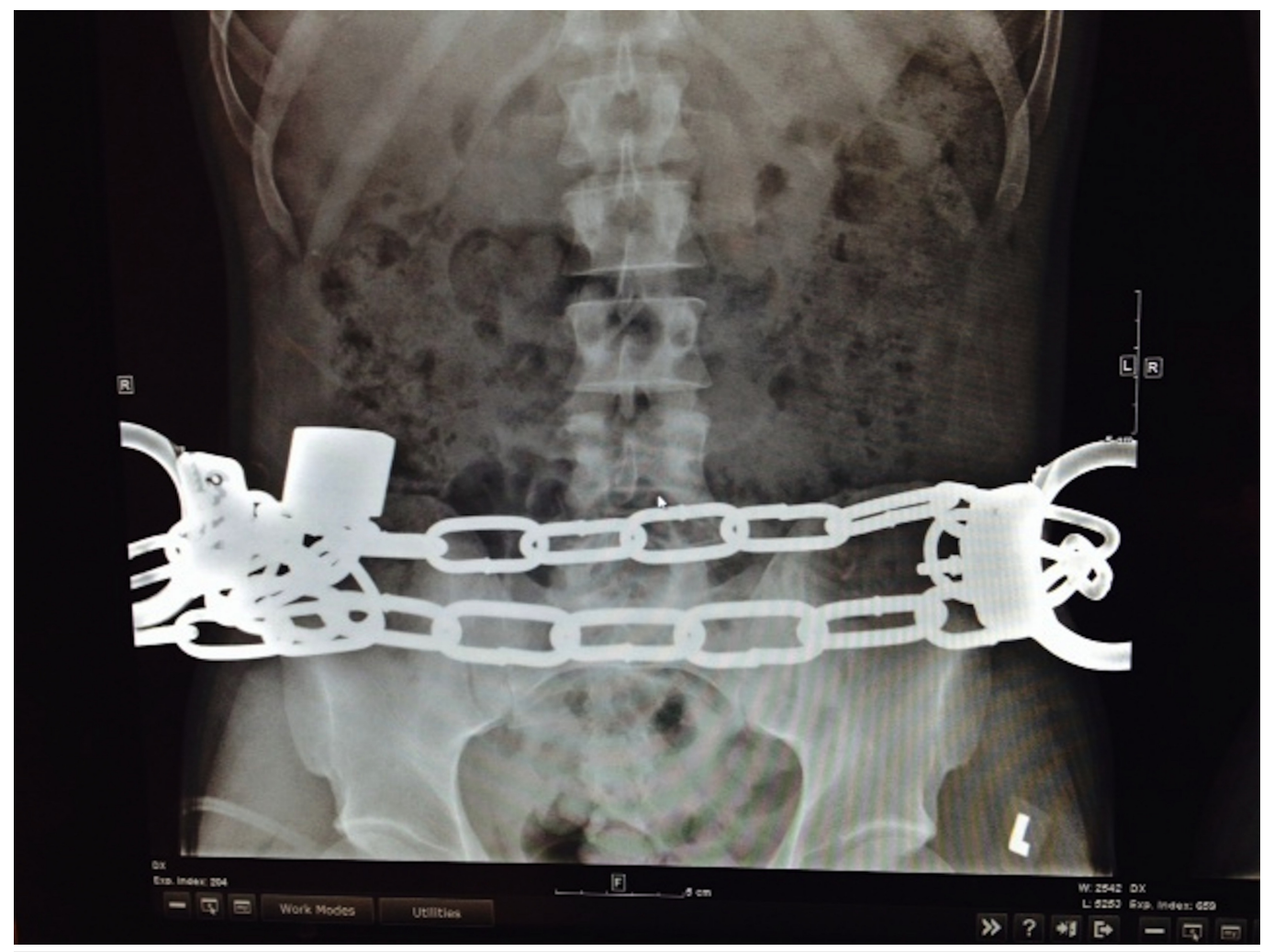

FIGURE 2: Normal abdominal x-ray image in hand-cuffed patient brought to Emergency from jail 


\section{Cureus}

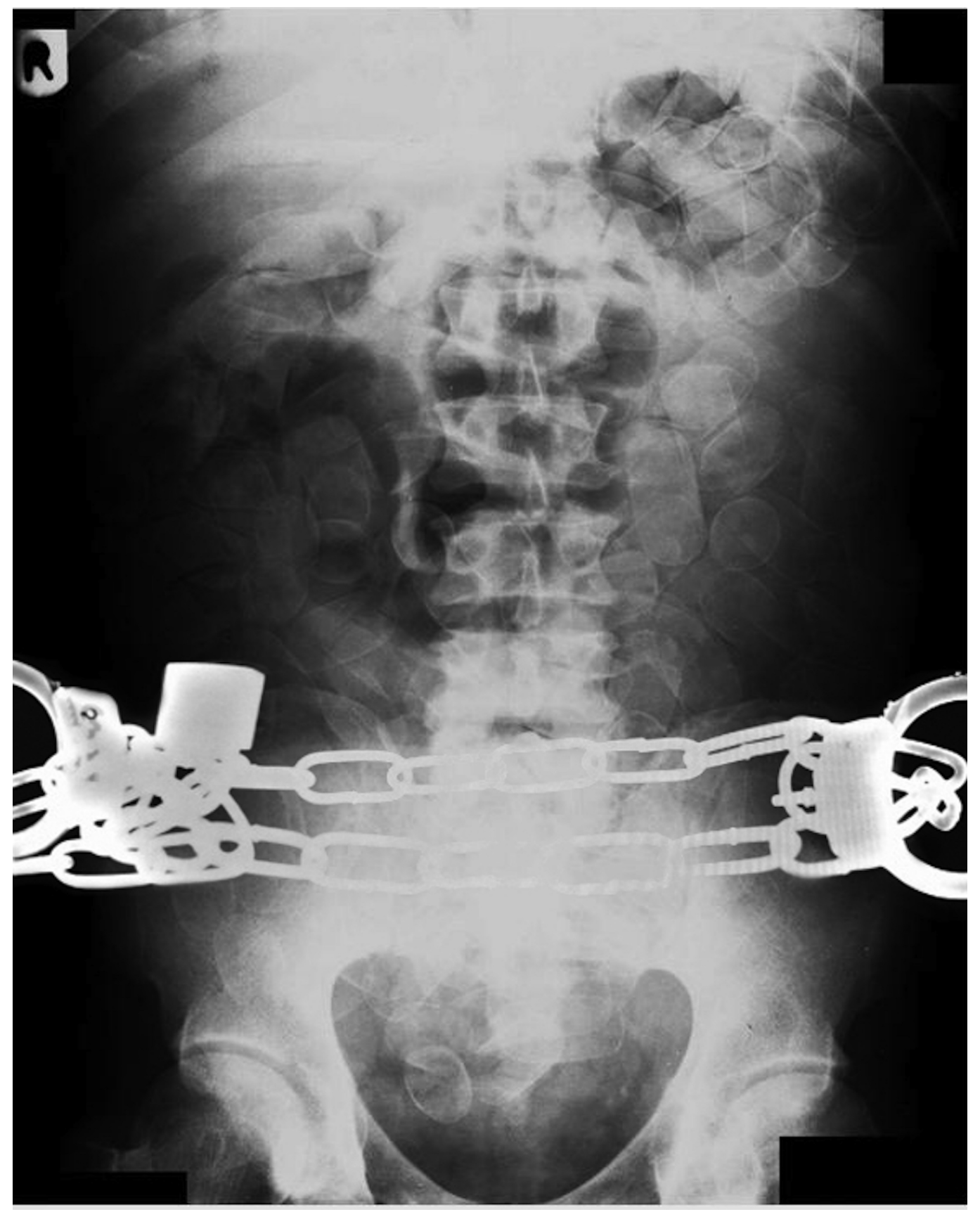

FIGURE 3: Synthesis of Figures 1 and 2, showing shackles as well as cocaine-filled packets in bowel

\section{Case}

The learning objectives for this simulation scenario are:

1. ABCs of resuscitation and forming an initial impression,

2. Recognition of the sympathomimetic toxidrome,

3. Recognition and treatment of cocaine toxicity while prioritizing its three most lethal complications: hyperthermia, hypertensive emergency, and cardiac arrhythmias, 


\section{Cureus}

\section{Recognition of body packing.}

In this simulation, the learners are informed that a young male, in this case, a Gaumard Noelle S575 human patient simulator, is brought in handcuffs to a tertiary care emergency room (ER), accompanied by two prison personnel. Trained Standardized Patients (SPs) act as the two prison personnel, who are present throughout the scenario to answer any questions (if relevant) but who are instructed not to provide any voluntary information. The learners are informed that the immigration authorities apprehended him from his Columbian flight when it landed unexpectedly in St. John's. They suspected drug trafficking because he paid cash for his oneway ticket, was wearing sunglasses at night, and seemed nervous. The patient is alert and well at the airport. His nose is running.

Table 1 describes the simulation algorithm used in progressively deteriorating the patient (Gaumard Noelle S575 human patient simulator) by the educator using the computer.

\section{Scenario}

You are an emergency room physician at a tertiary care center and have just begun your shift. A 26-year-old patient who is accompanied by law enforcement, is your first patient. On return from Columbia he was arrested at the airport on drug related charges. He is currently being triaged.

Begin Scenario - Learner enters the patient's room

\section{Objective 1: Airway, breathing, and circulation/Initial impression}

\section{Additional Scenario Data/ Physical Exam Findings Vital Signs Appropriate Learner Action}

Patient is in handcuffs and shackles. Patient is delirious and diaphoretic. He is sniffing and his nose is running.

Vital signs:

BP Immediately brings to resuscitation bay. While taking 180/110, history and physical, initiates 2 large bore iv's, cardiac HR 160, T monitor,ekg,finger stick glucose, thiamine $100 \mathrm{mg}$ iv, 38.8, RR dextrose.

24 GCS 15

\section{Objective 2: Recognition of sympathomimetic toxidrome}

\section{Additional Scenario Data/ Physical Exam Findings Vital Signs}

Teacher as penetentiary attendent: "We got this guy off a flight. Took him to the Pen 'cos he looked suspicious. Started to act strange in the jail so we brought him to you, Doc." If examined: The patient is very diaphoretic and agitated. Mydriasis and hyperreflexia are noted, abdomen is nontender, bowel sounds are decreased, lung sounds clear, equal air entry bilaterally, normal $\mathrm{S}_{1} \mathrm{~S}_{2}$

Laboratory/ECG/Diagnostic imaging results:
Vital signs: BP 180/110, HR 160, T 38.8, RR 24 GCS 15

\section{Appropriate Learner Action}

Orders: Complete blood count (CBC), electrolytes, BUN, creatinine, creatine kinase (CK), alcohol level, venous blood gas, serum osmolality, liver function panel(LFTs), troponin,INR, urinalysis/urine drug screen, blood cultures, ECG

If Ordered: Bedside glucose: 6 mmol/L ECG: Sinus tachycardia and diffuse ischemia: See Figure 4 Abdominal xray: pending

Objective 3: Recognition and treatment of cocaine toxicity, and priorizing its 3 most lethal complications: 


\section{Cureus}

\section{hyperthermia, hypertensive emergency and cardiac arrythmias}

Teacher as nurse: "Doctor, the blood work is drawn, but your patient is more agitated and is yelling. He is really diaphoretic now and he is grinding his teeth. $\mathrm{He}$ is muttering something about chest pain."

If no benzodiazapines ordered

If no cooling measures initiated

Patient says the chest pain is not so bad

Laboratory/ECG/Diagnostic imaging results

ECG: Na Blockade See Figure 5

If no management of arrhythmia

Absominal xray not yet available

Teacher as nurse: "Doctor the blood work is back"

Laboratory/ECG/Diagnostic imaging results:

Lab results as ordered by learner: Electrolytes: $\mathrm{K}-6.8 \mathrm{mmol} / \mathrm{L}$, Na 140 mmol/L, CBC: Normal LFT's: Normal PT/PTT: Normal Serum osmolality: $290 \mathrm{mmol} / \mathrm{kg}$ Creatinine: 140 umol/L CK: 5553 U/L Alcohol: negative pH: 7.29 Urine: hemoglobin positive/ microscopy negative for red blood cells OR myoglobin positive. Cocaine positive (may occur if small amounts drug ingested during ingestion of packets, or if packet ruptures)

Teacher as nurse: "Doctor the patients blood pressure is $190 / 130$ "

Vital signs:

BP 190/130, HR 120, T $37^{9}, \operatorname{RR} 24$

No change

Patient

If learner gives beta blockers
Vital signs:

BP 140/85,

$$
37^{9} \text {, RR } 22
$$

GCS 15

Vital Signs:

Learner reassesses the patient. Orders repeated doses of benzodiazepines. Simultaneously prepares for Rapid Sequence Intubation if airway not secure or patient's predicted clinical course mandates preemptive airway control. Initiates intensive cooling measures such as cool wet sheets, fans, ice packs to axillae and groins, or ice immersion in refractory hyperthermia. Aggressive hydration with crystalloid Nitroglycerine, benzodiazepines for chest pain

Learner continues to assess patient. Orders repeat ECG

Manages arrhythmia by reversing sodium blockade with sodium bicarbonate 1-2 meq/kg

Manages lab abnormalities. Continues aggressive hydration. 1 salbutamol neb,10 units regular insulin IV and $50 \mathrm{cc}$ of $50 \%$ dextrose IV. Consider iv calcium gluconate
Manages hypertension with increased doses benzodiazepines

Orders high-dose nitroglycerine for afterload reduction, nitroprusside or phentolamine when benzodiazepines ineffective. An advanced learner may suggest labetalol if they can explain the choice (alpha and beta blockade).

Vital signs: 


\section{Cureus}

$\begin{array}{lll}\text { Teacher as nurse: "Help, he is having a seizure" } & \text { BP } & \text { Manages with benzodiazepines endotracheal } \\ \text { 170/100, } & \text { intubation if airway compromised, or if indicated by } \\ \text { HR 120, T } & \text { predicted course of the patient } \\ 39, \text { RR } 24 & \end{array}$

\section{Objective 4: Recognition and treatment of body packing}

Upon recognition of the sympathomimetic toxidrome and the appropriate management of the most critical problems, the learner is informed that the patient continues to have unusually prolonged symptoms as if he continues to be exposed to the substance Vital signs: BP 160/92 HR 125, T $37^{9}$, RR 26 GCS 15

Laboratory/ECG/Diagnostic imaging results:

\section{X-ray: See Figure 3}

If no whole bowel irrigation ordered: Teacher as Police: "You know Doc, we were kind of

Vital signs concerned he might be a drug mule"

deteriorate
Appropriate treatment results in the simulated patient stabilizing
Learner considers the possibility of drug concealment/body packing. Abdominal xray now available.
If airway secure, learner consults surgery immediately for packet removal,considers oral charcoal, initiates whole bowel irrigation if time permits. Strongly considers endotracheal intubation to facilitate all of the above

End Scenario

\section{TABLE 1: Simulation Template}

Blood Pressure (BP) Heart Rate (HR) Temperature (T) Respiratory Rate (RR) Glasgow Coma Scale (GCS)

\section{Debriefing}

Debriefing has been described as a critical component of the simulation exercise [2]. Initially, students are encouraged to discuss their feelings about the case. To encourage candor, we allow a maximum of one teacher per learner. We use the advocacy-inquiry technique [3], which allows us to frame the trainee's thought processes non-judgmentally, and to neutrally suggest other approaches which might be more fruitful.

\section{Post-scenario didactics}

A brief didactic session, which addresses the learning objectives, is held after the debrief. This allows consolidation of the knowledge acquired during the simulation and closes any knowledge gaps.

\section{Discussion}

Drug muling was first described in Canada in 1973 [4], when a single condom filled with hashish caused a small bowel obstruction and was uneventfully removed.

Body packing with sympathomimetic illicit drugs is globally increasing in frequency [5], partly due to increased airport security post-September 11th [6], but it remains an uncommon problem in many small centres. Failure to recognize the presence of body packing can lead to 
potentially fatal medical errors. For example, the patient in our scenario was taken to jail from the airport when it may have been more appropriate to bring him to the ER.

Several kinds of drugs can be carried in the alimentary tract or vagina, including cocaine, heroin, ecstasy, and cannabis products. Typically, only one kind of drug is transported at a time. The amount of drug carried is variable; 50-200 packets, each containing up to $10 \mathrm{gm}$ of a drug, has been described. A single ruptured packet may cause death as more than 10 times the lethal dose of cocaine may be released [7]. Drug packets, once primitively constructed, are now precisely crafted with well-compacted contraband covered in latex and sometimes wax. They are uniform in appearance and occasionally wrapped in foil to alter their radiographic appearance [8]. Body packers usually know exactly how many packets they are carrying, but may deny carrying any. Luggage contents may include large numbers of condoms, latex fingers and gloves, petroleum jelly, coconut oil for swallowing packets, toilet paper, room deodorants, and coal packets, as well as medicines to control abdominal cramping and diarrhea. If body packers elude arrest, laxatives are taken to encourage packet expulsion; if they come to the hospital and remain asymptomatic, packets are counted when expelled, then given to police. Two consecutive normal stools indicate that the drug has passed [7].

Body packing with cocaine can be lethal. The condoms, balloons, or cellophane enclosing the drug can leak or rupture, causing a potentially fatal overdose. The packets themselves can cause a bowel obstruction, perforation, and/or peritonitis. Esophageal rupture can occur. As one leaking packet of drug can release a fatal amount of cocaine, it is important for emergency medicine trainees to recognize the sympathomimetic toxidrome, the other toxicities caused by cocaine, and the presence of body packing, which implies that many more packets of drug are concealed inside the body where they can leak and worsen the toxicity. In a stable patient, any free drug will be absorbed somewhat by oral charcoal, while the packets are expelled over time with whole bowel irrigation. Should symptoms and signs of cocaine toxicity develop, surgery must be consulted while resuscitation is underway for packet removal via laparotomy. Laparoscopic removal risks tearing the condoms. Conservative management is usually successful when the packets are not leaking [9-10].

This simulation scenario uses a computerized human mannequin to teach emergency medicine trainees an approach to the sympathetic toxidrome, cocaine toxicity, and body packing. It also uses a "fiction within a fiction": two real abdominal x-ray images are superimposed to create a third entity which helps tell the story. Because of the reports of controversy about the ethical difference between improving an image, and frankly altering it [11], we have decided to acknowledge this fact and admit to the factitious nature of the image while discussing our contract with the learner. In order to avoid disclosing details prematurely, the images themselves are not shown until later in the simulation. The x-ray depicting shackles and handcuffs in an otherwise unremarkable film came from an actual patient from a local jail. Usually shackles and cuffs are not imaged because the patient raises their hands above their head; on this occasion, an error in our x-ray department led to the inclusion of the hardware. We felt that such a striking and unusual image could form the basis for our body packing scenario. Since we rarely see body packing, and have no actual images of it from our own population, we acquired the x-ray showing packets within the bowel online and superimposed the two. The resulting image showing shackles and handcuffs as well as packets in the bowel, may be "larger than life", but it serves to illustrate the diagnosis in our setting, where body packing is rare now, but potentially will become more frequent.

As mentioned earlier, the learning objectives for this simulation scenario were:

1. ABCs of resuscitation and forming an initial impression, 
2. Recognition of the sympathomimetic toxidrome,

3. Recognition and treatment of cocaine toxicity while priorizing its three most lethal complications: hyperthermia, hypertensive emergency, and cardiac arrythmias,

4. Recognition of body packing.

These objectives were developed because cocaine toxicity produces a multi-system assault. Hyperthermia must be promptly and aggressively managed with cooling techniques and benzodiazepines in order to reduce mortality. It is associated with rhabdomyolysis, hyperkalemia (which may worsen cardiac arrhythmias), renal failure, and myocardial ischemia (Figure 4). Hypertensive emergencies associated with cocaine overdose are treated with benzodiazepines and phentolamine (a pure alpha-blocker), and high-dose nitroglycerine or nitroprusside. Beta-blockade will worsen the situation. Cardiac arrhythmias seen are most commonly sinus tachycardia, but excess adrenalization may produce atrial fibrillation (AF) or supraventricular tachycardia (SVT). Wide-complex ventricular tachycardia may happen in association with fast sodium channel blockade (Figure 5) and is treated with sodium bicarbonate. It may be preceded by the RSR' pattern in AVR, as in other sodium channel blockers overdoses, such as tricyclic antidepressants. Lidocaine and other $1 \mathrm{~A}$ anti-arrhythmics must be avoided because they block sodium channels. The presence of body packing raises the possibility of worsening toxicity as well as mechanical bowel obstruction, perforation, and peritonitis.

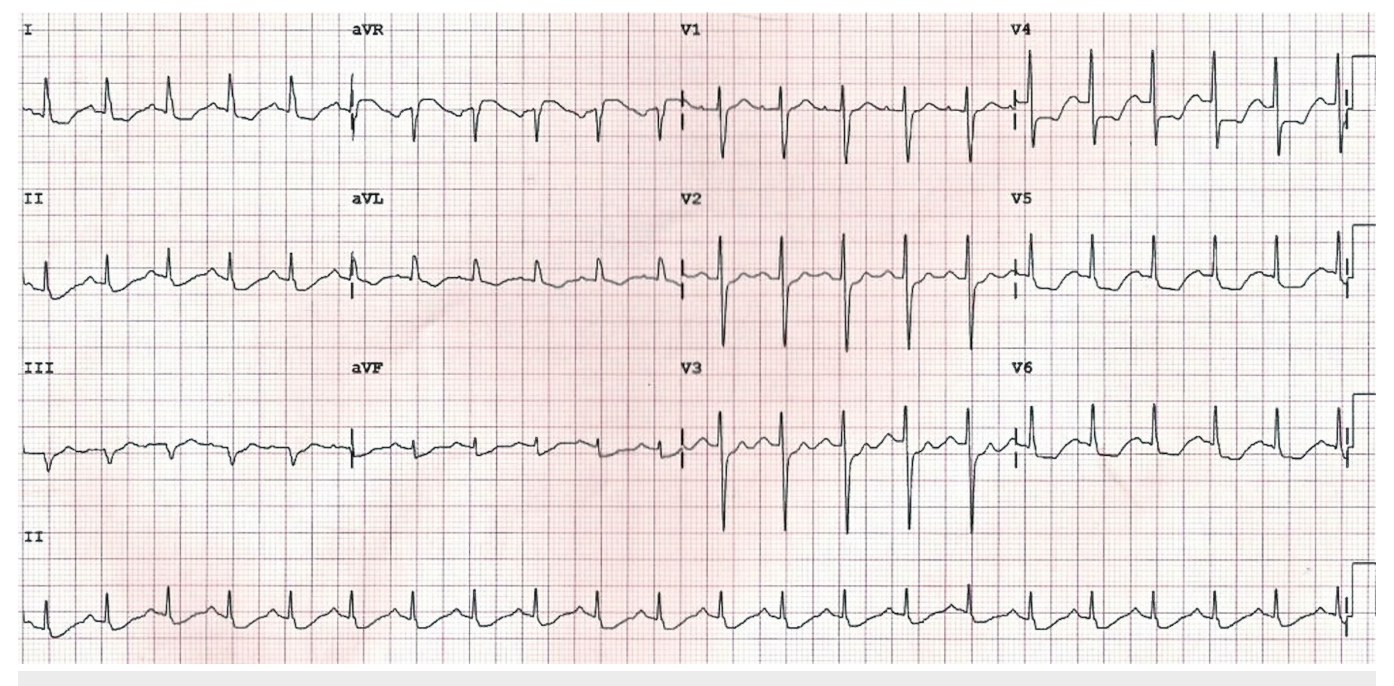

FIGURE 4: Diffuse ischemic change

Commonly seen with chest pain in cocaine intoxication [12] 


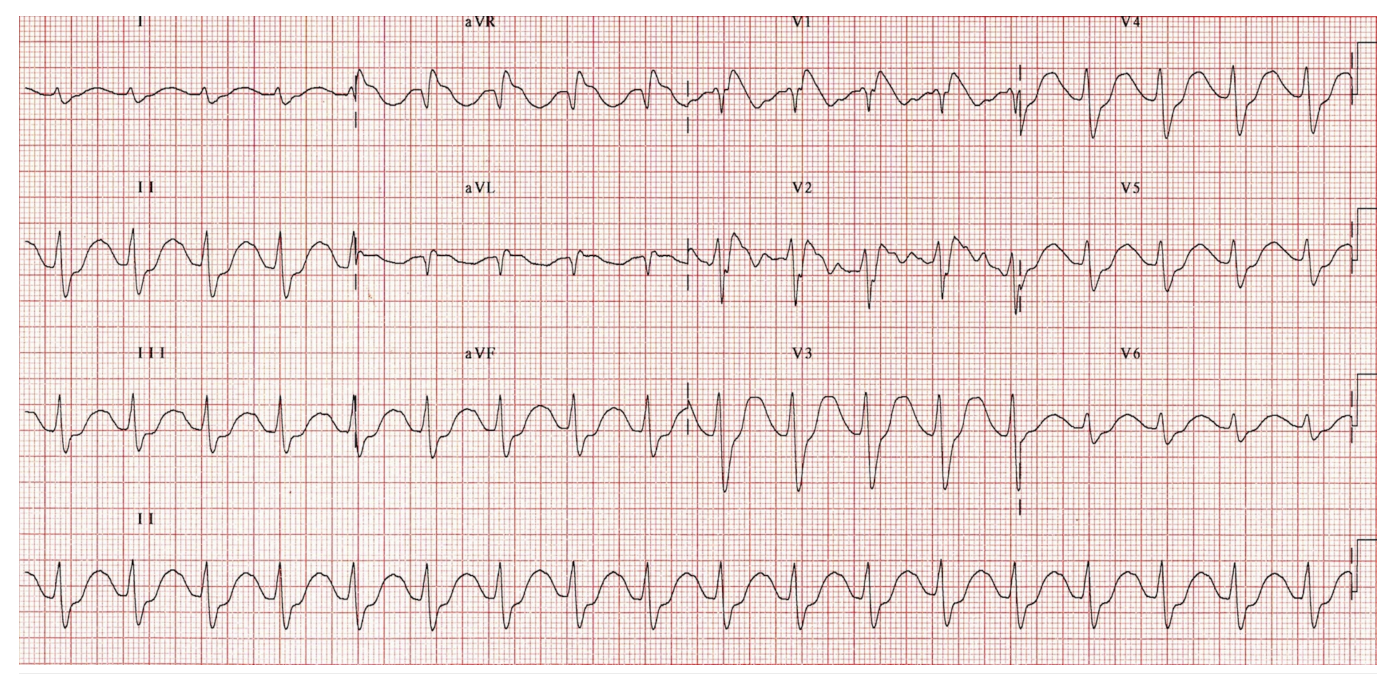

FIGURE 5: RSR' pattern and wide QRS typical of sodium channel blockade.

Seen in cocaine and other toxicities like tricyclic anti-depressants [13].

Patients with cocaine toxicity often present with toxic delirium, in which it is important to consider other causes, such as infection, Wernieke's encephalopathy, or trauma. It is prudent to order blood cultures, if indicated, and administer thiamine and dextrose at the outset.

\section{Conclusions}

Simulation is a valuable tool for teaching emergency medicine trainees the effects of cocaine toxicity in the context of body packing. Simulation includes a fictional contract in which teachers and learners acknowledge that the case in question is not real, but agree to suspend their disbelief and proceed as though it is. We describe a simulation exercise in which the transposition of two real x-ray images produces a third image, which helps tell the story.

\section{Additional Information}

\section{Disclosures}

Human subjects: All authors have confirmed that this study did not involve human participants or tissue. Animal subjects: All authors have confirmed that this study did not involve animal subjects or tissue. Conflicts of interest: In compliance with the ICMJE uniform disclosure form, all authors declare the following: Payment/services info: All authors have declared that no financial support was received from any organization for the submitted work. Financial relationships: All authors have declared that they have no financial relationships at present or within the previous three years with any organizations that might have an interest in the submitted work. Other relationships: All authors have declared that there are no other relationships or activities that could appear to have influenced the submitted work.

\section{Acknowledgements}

This project was supported by Tuckamore Simulation Research Collaborative and Emergency Medicine Education Committee, Memorial University of Newfoundland. Two original x-ray images were combined digitally to create a third image. Thanks to Luke Merdsoy for this work. 


\section{References}

1. Issenberg SB, Scalese RJ: Simulation in health care education. Perspect Biol Med. 2008, 51:3146. 10.1353/pbm.2008.0004

2. Rudolph JW, Simon R, Rivard P, Dufresne RL, Raemer DB: Debriefing with good judgment: Combining rigorous feedback with genuine inquiry. Anesthesiol Clin. 2007, 25:361-76.

3. Helminski L, Koberna S: Total quality in instruction: A systems approach . Academic initiatives in total quality for higher education. Roberts HV (ed): ASQC Quality Press, Milwaukee, WI; 1995. 309-362.

4. Deitel M, Syed AK: Intestinal obstruction by an unusual foreign body . Can Med Assoc J. 1973, 109:211-2.

5. Hergan K, Kofler K, Oser W: Drug smuggling by body packing: What radiologists should know about it. Eur Radiol. 2004, 14:736-42.

6. Stampede of drug mules at Kennedy: after 9/11 lull, heroin \& ecstasy busts soar . (2002). Accessed: September 17, 2014: http://www.nydailynews.com/archives/news/stampede-drugmules-kennedy-9-11-lull-heroin-ecstasy-busts-soar-article-1.48....

7. Rao RB, Hoffman RS: Cocaine, amphetamines, and other sympathomimetics. Rosen's emergency medicine: concepts and clinical practice. Mosby, St. Louis, MO; 2002. 1994-2000.

8. Traub SJ, Hoffman RS, Nelson LS: Body packing--the internal concealment of illicit drugs . NEJM. 2003, 349:2519-26.

9. Beckley I, Ansari NA, Khwaja HA, Mohsen Y: Clinical management of cocaine body packers: the Hillingdon experience. Can J Surg. 2009, 52:417-21.

10. Bulstrode N, Banks F, Shrotria S: The outcome of drug smuggling by 'body packers'--the British experience. Ann R Coll Surg Engl. 2002, 84:35-8.

11. Bourne PE, Barbour V: Ten simple rules for building and maintaining a scientific reputation . PLoS Comput Biol. 2011, 7:e1002108. 10.1371/journal.pcbi.1002108

12. Tricyclic overdose (Sodium-Channel Blocker Toxicity) . (2014). Accessed: September 17, 2014 : http://lifeinthefastlane.com/ecg-library/basics/tca-overdose.

13. Myocardial Ischaemia. (2014). Accessed: September 17, 2014: http://lifeinthefastlane.com/ecg-library/myocardial-ischaemia/. 